\title{
Cluster-shape Based Improvement of Spatial Resolution for the Belle II DEPFET Pixel Detector
}

\author{
Peter Kodys* \\ Charles University in Prague, on behalf of the DEPFET collaboration \\ E-mail: peter.kodys@mff.cuni.cz
}

\begin{abstract}
A pixel detector built with the DEPFET technology will be used for the two innermost layers of the Belle II detector at the $\mathrm{e}^{+} \mathrm{e}^{-}$SuperKEKB collider. The physics goals of the experiment impose challenging requirements to the design of the pixel detector in terms of performance, material budget and power consumption. The DEPFET technology has proven to be a suitable solution for the Belle II requirements and has been chosen as the baseline for the detector. This paper reviews the DEPFET pixel detector for Belle II and the various system aspects that have driven its final design with special focus on cluster-shape based improvement of spatial resolution for the detector.
\end{abstract}

The 23rd International Workshop on Vertex Detectors

15-19 September 2014

Macha Lake, The Czech Republic

\footnotetext{
* Speaker.
} 


\section{Introduction}

The SuperKEKB collider, an upgrade of the former KEKB, is under construction at the site of the High Energy Accelerator Research Organization (KEK) in Tsukuba, Japan. It is an asymmetric ( $4 \mathrm{GeV}, 7 \mathrm{GeV}) \mathrm{e}^{+} \mathrm{e}^{-}$collider working at the center-of-mass energy of the $\Upsilon(4 S)$ resonance. The design peak luminosity is $8 \times 10^{35} \mathrm{~cm}^{-2} \mathrm{~s}^{-1}$, about 40 times larger than KEKB, aiming at an integrated luminosity of $50 \mathrm{ab}^{-1}[1]$.

To fully exploit the higher luminosity, the former Belle detector is being upgraded. The new detector must cope with higher backgrounds and event rates, and the corresponding larger radiation damage, occupancy and fake hit production. As a consequence, improved vertexing and tracking capabilities are needed in the Belle II detector. This makes the vertex detector one of the key elements that drive the physics performance. It consists of four layers of double-sided silicon strip detectors in the outer radii, SVD, and two layers of highly granular pixel sensors in the innermost part, known as PXD.

\section{DEPFET sensors at Belle II}

The Belle II experiment requires excellent vertexing performance in the vicinity of the primary interaction region. The DEPFET technology of active pixel sensors is among the frontier detector concepts for high energy physics at high luminosity [2]. The PXD is intended to improve vertex resolution compare to Belle. As a consequence of low momentum of some particles in the final state $(<1 \mathrm{GeV} / \mathrm{c})$, the hit position determination is intrinsically limited due to multiple Coulomb scattering. This sets a lower limit of $10 \mu \mathrm{m}$ for the spatial resolution in the PXD that can be achieved with a moderate pixel size of $50 \times 55 \mu \mathrm{m}^{2}$ upto $50 \times 85 \mu \mathrm{m}^{2}$. This pixel size is also enough to cope with the expected occupancy of $0.4 \mathrm{hits} \mu \mathrm{m}^{-2} \mathrm{~s}^{-1}$. For the same reason the material budget must be kept low, up to a maximum of $\sim 0.2 \% \mathrm{X}_{0}$ per layer, implying a thickness of $75 \mu \mathrm{m}$ of the sensitive part of the sensor. The acceptance of the detector must cover the range $17^{\circ}-155^{\circ}$ in azimuth angle.

The detector will be read continuously with a frame time of $20 \mu$ s keeping the occupancy below $3 \%$. This continuous readout means that charge collecting is ON all the time, which together with the restrictions on material budget sets the requirements for cooling. The in-pixel amplification of the DEPFET technology allows for 75 micron thin low-noise sensors which do not require additional support or cooling structures in the active region of the detector. Finally, according to the simulations, the radiation dose expected in the inner region of the detector is around $2 \mathrm{Mrad} / \mathrm{yr}$.

A unique gated data acquisition mode allows to suspend data readout for time periods with high noise occupancy; over such periods, acquired charge is frozen and kept for later readout. The front end electronics and the data acquisition schemes supporting the integration into Belle II are finalized and the two-layer pixel vertex detector will be ready for data acquisition from its 7.6 million pixels in 2016 (see also Fig. 1).

\section{Design considerations}

Current experiments use pixel detectors placed as close as possible to the collider interaction point. Such detectors face several design challenges: they have to combine low mass for non- 


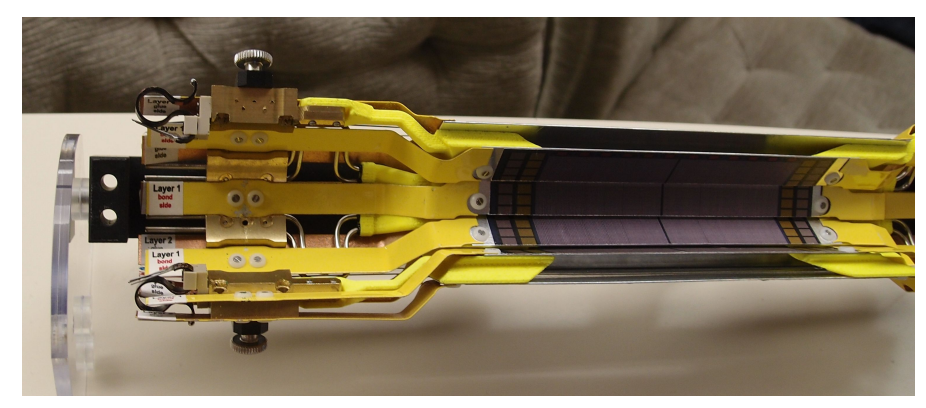

Figure 1: PXD for Belle II - design and mockup by MPI Munich.

intrusiveness with high granularity for high vertexing performance at high beam background exposure. The high granularity and beam background lead to huge data flows from the innermost layers, creating additional constraints related to reasonably achievable transmission and data processing bandwidths.

A compromise between data flow and tracking/vertexing performance has to be sought, as well as improvements in noise suppression and data reconstruction that allow to push design optima within achievable limits. Increased charge-sharing between pixels is a typical example, allowing to increase pixel size and, consequently, decrease data flows, while keeping spatial resolution of a sensor.

The DEPFET technology can produce matrices with a wide range of pixel sizes, from 20 microns to over 100 microns. Pixel sizes can even be optimized within sensor ladders to further reduce granularity and data flows. Some basic data are collected in table 1.

\begin{tabular}{ccc} 
& Inner Layer & Outer Layer \\
\hline Modules & 8 & 12 \\
\hline Thickness & 75 microns & 75 microns \\
\hline Length Ladder & $90 \mathrm{~mm}$ & $123 \mathrm{~mm}$ \\
\hline Sensitive Sensor & $44.8 \times 12.5 \mathrm{~mm}^{2}$ & $61.44 \times 12.5 \mathrm{~mm}^{2}$ \\
\hline Pixel Size & $55 / 60 \times 50 \mu \mathrm{m}^{2}$ & $70 / 85 \times 50 \mu \mathrm{m}^{2}$ \\
\hline Pixels & $3.072 \times 10^{6}$ & $4.608 \times 10^{6}$ \\
\hline Frame Rate & $50 \mathrm{kHz}$ & $50 \mathrm{kHz}$ \\
\hline
\end{tabular}

Table 1: Summary table of DEPFET sensor parameters in the Belle II experiment.

\section{Data acquisition}

With 40 half-ladders (Fig. 2) of $250 \times 768$ pixels, PXD has $7.6 \times 10^{6}$ pixels. At expected occupancy of up $3 \%$ and trigger rate of $30 \mathrm{kHz}$ it produce $180 \mathrm{~GB}$ raw data per second. Belle II PXD produces $180 \mathrm{~GB} / \mathrm{s}$ of raw data. Forty PXD half ladders (Fig. 2) with $250 \times 768$ pixels each and $7.6 \times 10^{6}$ pixels in total, expected occupancy up to $3 \%$, trigger rate of $30 \mathrm{kHz}$. To reach achievable data flow, data volume must be reduced by at least a factor of 10 before the PXD stream is combined with data from other subdetectors. 


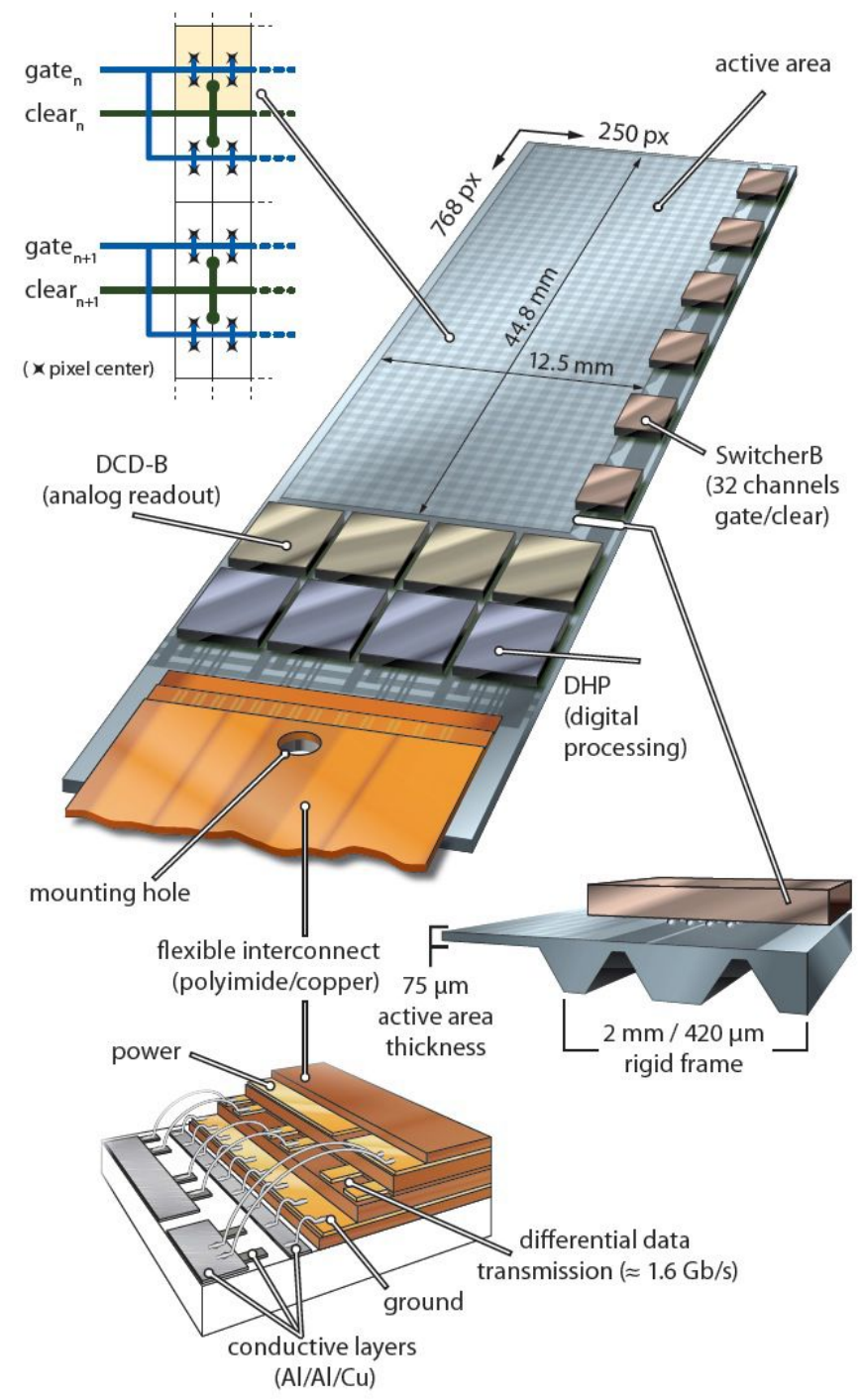

Figure 2: Design and readout structures of DEPFET half-ladder.

PXD module readout [3] contains Gate and Clear Switchers to organize the readout sequence, DCD (Drain Current Digitizer) are readout chips and A/D converters, DHP (Digital Handling Processor) chips provide first-stage pre-processing and data reduction (pedestals, common mode noise correction, zero suppression, compression algorithm). Signals continue from DHP to DHH (Data Handling Hybrid) via $15 \mathrm{~m}$ long kapton with converted to twisted-pair in a passive patch panel, from DHHs via optical links to ATCA Compute Nodes (ATCA CNs). ATCA CNs reduce the data based on triggers and allow for fast tracking using SVD data to quickly identify regions of interest in the PXD layers. 


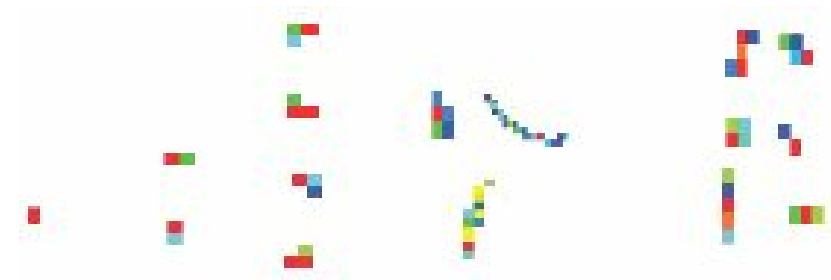

Figure 3: Categorization of cluster shapes: 1-pixel, 2-pixels, 3-pixels, non-symmetrical, symmetrical.

\section{Hit position reconstruction}

Several methods are available to improve hit position reconstruction in the Belle II vertex detector. The point where a particle track crosses a sensor is reconstructed using pixel charges in a cluster, RMS pixel noises and clustering thresholds. These data are used to estimate hit position and its uncertainty.

1. Sensor design provides efficient charge transport from the full bulk to internal gate of DEPFET.

2. Ladders are tilted to increase charge sharing between pixels in R- $\phi$ coordinate.

3. Correction of position bias of reconstructed cluster position for non-symmetric small clusters.

\section{Using cluster shape to improve reconstruction of hit position estimates}

Cluster shape analysis is based on full simulation of the PXD in the basf2 framework (simulation and reconstruction framework developed for the Belle II experiment). The accuracy and precision of hit position estimates strongly depends on cluster size. There are five basic types of clusters (Fig. 3): single, double and triple pixel clusters, and the rest of symmetrical and nonsymmetrical clusters. For a mixture of electrons, positrons and pions with energies of $0.05-3.0 \mathrm{GeV}$, shot from Belle II interaction point uniformly in R- $\phi$ and $\theta$ of $17^{\circ}-150^{\circ}$, noise $\sigma$ set to $300 \mathrm{ENC}$, $25 \%$ form single-pixel clusters, $15 \%$ form 2-pixel clusters along the R- $\phi$ coordinate, $26 \% 2$ pixel clusters along the z-coordinate, $12 \%$ form non-symmetric "L"-shaped three-pixel clusters, $16 \%$ form larger non-symmetrical clusters, and the rest $6 \%$ form symmetrical clusters (like $2 \times 2$ clusters). The dependence of the cluster shape types on cluster cuts is shown in Fig. 4.

For single-pixel clusters, the obvious hit position estimate is the center of the pixel. For larger clusters, hit position is estimated separately for the u-coordinate (R- $\phi)$ and $\mathrm{v}$ - coordinate (z-direction), using center-of-gravity estimates for clusters size 2 and the analog head-tail method for size 3 and more. Generally, the average resolution is best for small clusters of size 2 and 3 .

For one-pixel clusters, hit position uncertainty is given by the area where a given energy deposition is mostly contained within the single pixel - it therefore depends on pixel charge and clustering threshold.

With particles arriving at different (and unknown) directions, the standard eta-correction algorithms are not usable. Therefore, simple bias-correcting methods for the center-of-gravity and 


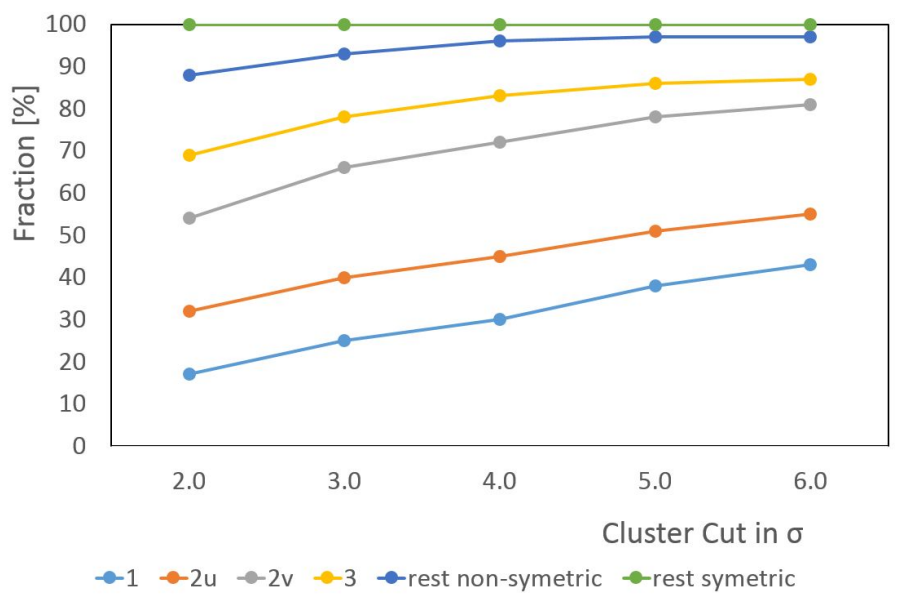

Figure 4: Fractions of cluster shape types for different clustering cuts $(\sigma=300$ ENC).
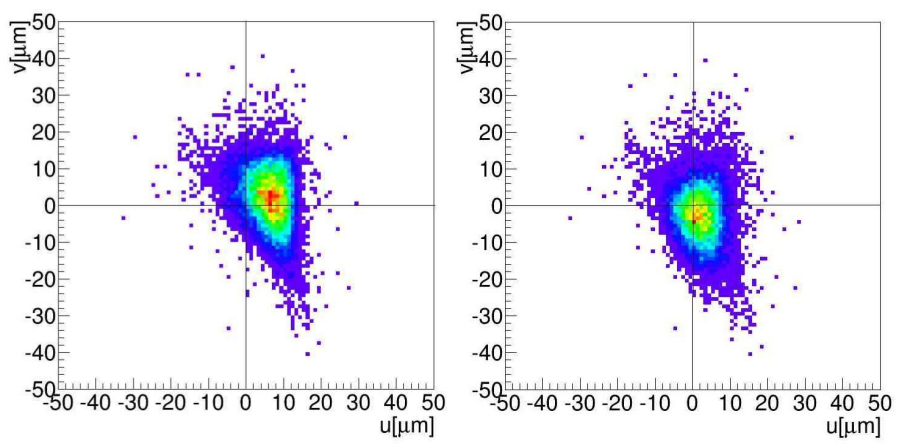

Figure 5: Residual plot of "L" shape 3-pixel clusters (in one "L" orientation) before (left) and after (right) correction.

head-tail estimates are desirable, that would only use measurable quantities to correct for the bias. The 3-pixel "L"-shaped clusters are the simplest and most common case where such a bias correction would be desirable. For these clusters, the center-of-gravity estimate is biased by about $10 \%$ of pixel size, comparable to the typical RMS error of the position estimate.

We show that adding a fixed (pixel noise dependent) charge to the pixel with zero signal in the $2 \times 2$ matrix can significantly improve the center-of-gravity estimate of hit position for such clusters. Adding the fourth pixel with the signal of $1.3 \times \sigma$ improved position RMS error from 7.4 microns to 4.7 microns in R- $\phi$, and reduced position bias from 5 to 2 microns. Bias and RMS error for different cuts and corrections are summarized on Tables 2 and 3.

\section{Conclusion}

The Belle II experiment is under construction at KEK. The goal of the first physics run in 2016 seems realistic and the pixel detector will be ready with all specifications met. Improvement of hit position reconstruction is important for vertexing performance and new physics searches. Realistic 

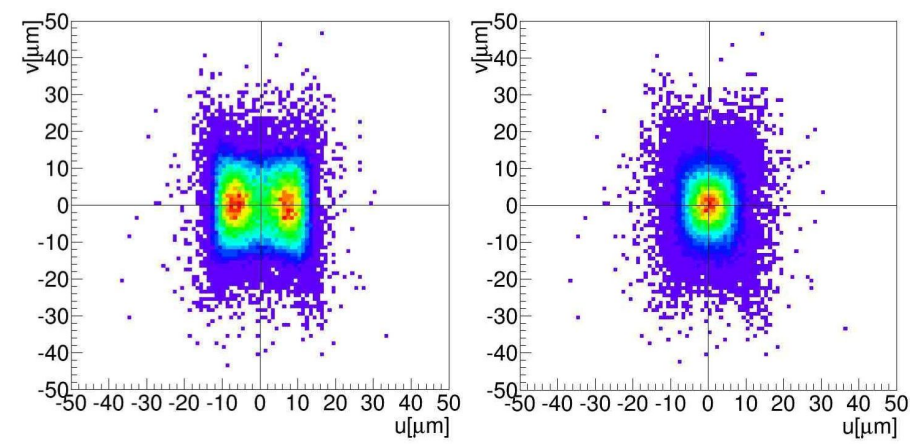

Figure 6: Residual plot of "L" shape 3-pixel clusters in sum of all "L" orientations before (left) and after (right) correction.

\begin{tabular}{cccccc} 
Cluster cut $[\sigma]$ & 2 & 3 & 4 & 5 & 6 \\
\hline No correction R- $\phi$ & 5.8 & 5.7 & .6 & 5.6 & 5.5 \\
\hline No correction in z & 4.0 & 3.4 & .1 & 2.9 & 3.0 \\
\hline Correction R- $\phi$ & 1.9 & .0 & 2.0 & 1.8 & \\
\hline Correction Z & -0.6 & -1.3 & -1.4 & -1.9 & \\
\hline
\end{tabular}

Table 2: Table for hit position bias (in microns) reconstructed from "L"-shaped clusters before and after correction for different clustering cuts $(\sigma=300 \mathrm{ENC})$.

\begin{tabular}{cccccc} 
Cluster cut $[\sigma]$ & 2 & 3 & 4 & 5 & 6 \\
\hline No correction R- $\phi$ & 7.6 & 7.4 & 7.4 & 7.4 & 7.5 \\
\hline No correction Z & .3 & 7.6 & 8.0 & 9.2 & 10.1 \\
\hline Correction $\mathrm{R}-\phi$ & 4.9 & 4.7 & 4.7 & 4.7 & \\
\hline Correction $\mathrm{Z}$ & 6.6 & 7.4 & 8.0 & 9.6 & \\
\hline
\end{tabular}

Table 3: Table for RMS error (in microns) reconstructed from "L"-shaped clusters before and after correction for different clustering cuts $(\sigma=300 \mathrm{ENC})$.

estimates of hit position uncertainties and correction of reconstruction bias is equally important. We have shown that the most important reconstruction biases in small "L"-shaped 3-pixel clusters can be easily corrected by adding a constant charge in the missing pixel. This efficiently corrects for center-of-gravity bias and improves the distribution of residuals. Impact on physics analyses is under study.

\section{Acknowledgments}

This research has been supported by the Czech Grant Agency under grant no. P203/10/0777, by the European Commission within the Framework Programme 7 Capacities, Grant Agreement 262025 and by the Ministry of Education, Youth and Sports of the Czech Republic under the project Nr. 7E12050. 


\section{References}

[1] Edited by: Z. Doležal and S. Uno, Belle II Technical Design Report, High Energy Accelerator Research Organization, KEK Report 2010-1 (2010)

arXiv:1011.0352v1 [physics.ins-det]

[2] M. Vos at al, DEPFET active pixel detectors for a future linear $e^{+} e^{-}$collider, TNS 6 No. 1 (2010) arXiv:1212.2160v1 [physics.ins-det]

[3] H. Kruger for the DEPFET Collaboration, Front-end electronics for DEPFET pixel detectors at SuperBelle(BELLE II), NIMA 617 (2010) 337-341 